\title{
Common fixed point theorems for generalized expansive mappings in partial $b$-metric spaces and an application
}

\author{
Chuanxi Zhu, Wenqing Xu*, Chunfang Chen and Xiaozhi Zhang
}

\section{"Correspondence:}

wen_qing_xu@163.com

Department of Mathematics,

Nanchang University, Nanchang,

330031, P.R. China

\begin{abstract}
In this paper, we first introduce the concepts of generalized $(\psi, f)_{\lambda}$-expansive mappings and generalized $(\phi, g, h)_{\lambda}$-weakly expansive mappings designed for three mappings. Then we establish some common fixed point results for such two new types of mappings in partial $b$-metric spaces. These results generalize and extend the main results of Karapınar et al. (J. Inequal. Appl. 2014:22, 2014), Nashine et al. (Fixed Point Theory Appl. 2013:203, 2013) and many comparable results from the current literature. Moreover, some examples and an application to a system of integral equations are given here to illustrate the usability of the obtained results.
\end{abstract}

MSC: $47 \mathrm{H} 10 ; 54 \mathrm{H} 25$

Keywords: partial metric space; $b$-metric space; expansive mappings; weakly expansive mappings; common fixed point

\section{Introduction and preliminaries}

Fixed point theory in metric spaces is an important branch of nonlinear analysis, which is closely related to the existence and uniqueness of solutions of differential equations and integral equations.

There are many generalizations of the concept of metric spaces in the literature. In particular, Matthews [1] introduced the concept of a partial metric space as a part of the study of denotational data for networks and proved that the Banach contraction mapping theorem can be generalized to the partial metric context for applications in program verification. After that, fixed point results in partial metric spaces have been studied by many authors (see [2-7]). On the other hand, the concept of a $b$-metric space was introduced and studied by Bakhtin [8] and Czerwik [9]. Since then, several papers have been published on the fixed point theory of the variational principle for single-valued and multi-valued operators in $b$-metric spaces (see [8-15] and the references therein). We begin with the definition of $b$-metric spaces.

Definition 1.1 ([8]) Let $X$ be a nonempty set and $\lambda \geq 1$ be a given real number. A function $d: X \times X \rightarrow R^{+}$is said to be a $b$-metric on $X$ if, for all $x, y, z \in X$, the following conditions are satisfied:

$\left(\mathrm{b}_{1}\right) d(x, y)=0$ if and only if $x=y$, 
$\left(\mathrm{b}_{2}\right) d(y, x)=d(x, y)$

$\left(\mathrm{b}_{3}\right) d(x, z) \leq \lambda[d(x, y)+d(y, z)]$.

In this case, the pair $(X, d)$ is called a $b$-metric space.

Recently, Shukla [16] introduced the notion of a partial $b$-metric space as a generalization of partial metric spaces and $b$-metric spaces.

Definition $1.2([16])$ Let $X$ be a nonempty set and $\lambda \geq 1$ be a given real number. A mapping $p_{b}: X \times X \rightarrow R^{+}$is said to be a partial $b$-metric on $X$ if for all $x, y, z \in X$, the following conditions are satisfied:

$\left(\mathrm{p}_{b 1}\right) p_{b}(x, x)=p_{b}(y, y)=p_{b}(x, y)$ if and only if $x=y$,

$\left(\mathrm{p}_{b 2}\right) p_{b}(x, x) \leq p_{b}(x, y)$

$\left(\mathrm{p}_{b 3}\right) p_{b}(x, y)=p_{b}(y, x)$,

$\left(\mathrm{p}_{b 4}\right) p_{b}(x, z) \leq \lambda\left[p_{b}(x, y)+p_{b}(y, z)\right]-p_{b}(y, y)$.

A partial $b$-metric space is a pair $\left(X, p_{b}\right)$ such that $X$ is a nonempty set and $p_{b}$ is a partial $b$-metric on $X$. The number $\lambda \geq 1$ is called the coefficient of $\left(X, p_{b}\right)$.

In [17], Mustafa et al. introduced a new concept of partial $b$-metric by modifying Definition 1.2 in order to guarantee that each partial $b$-metric $p_{b}$ can induce a $b$-metric. The advantage of the new definition of partial $b$-metric is that by using it one can define a dependent $b$-metric which is called the $b$-metric associated with partial $b$-metric $p_{b}$. The new concept of partial $b$-metric is as follows.

Definition 1.3 ([17]) Let $X$ be a nonempty set and $\lambda \geq 1$ be a given real number. A mapping $p_{b}: X \times X \rightarrow R^{+}$is said to be a partial $b$-metric on $X$ if for all $x, y, z \in X$, the following conditions are satisfied:

$\left(\mathrm{p}_{b 1}\right) p_{b}(x, x)=p_{b}(y, y)=p_{b}(x, y)$ if and only if $x=y$,

$\left(\mathrm{p}_{b 2}\right) p_{b}(x, x) \leq p_{b}(x, y)$

$\left(\mathrm{p}_{b 3}\right) p_{b}(x, y)=p_{b}(y, x)$,

$\left(\mathrm{p}_{b 4}^{\prime}\right) \quad p_{b}(x, z) \leq \lambda\left[p_{b}(x, y)+p_{b}(y, z)-p_{b}(y, y)\right]+\frac{(1-\lambda)}{2}\left[p_{b}(x, x)+p_{b}(z, z)\right]$.

The pair $(X, b)$ is called a partial $b$-metric space with coefficient $\lambda \geq 1$.

Since $\lambda \geq 1$, from $\left(\mathrm{p}_{b 4}^{\prime}\right)$, we have

$$
p_{b}(x, z) \leq \lambda\left[p_{b}(x, y)+p_{b}(y, z)-p_{b}(y, y)\right] \leq \lambda\left[p_{b}(x, y)+p_{b}(y, z)\right]-p_{b}(y, y) .
$$

Hence, a partial $b$-metric in the sense of Definition 1.3 is also a partial $b$-metric in the sense of Definition 1.2.

In a partial $b$-metric space $\left(X, p_{b}\right)$, if $p_{b}(x, y)=0$, then $\left(\mathrm{p}_{b 1}\right)$ and $\left(\mathrm{p}_{b 2}\right)$ imply that $x=y$. But the converse does not hold always. It is clear that every partial metric space is a partial $b$-metric space with coefficient $\lambda=1$ and every $b$-metric is a partial $b$-metric space with same coefficient and zero distance. However, the converse of these facts need not hold. The following example shows that a partial $b$-metric on $X$ might be neither a partial metric, nor a $b$-metric on $X$. 
Example 1.1 ([17]) Let $X=R, q>1$ be a constant and $p_{b}: X \times X \rightarrow R^{+}$be defined by

$$
p_{b}(x, y)=|x-y|^{q}+3
$$

for all $x, y \in X$. Then $\left(X, p_{b}\right)$ is a partial $b$-metric space with the coefficient $\lambda=2^{q-1}>1$, but it is neither a $b$-metric nor a partial metric space.

Each partial $b$-metric $p_{b}$ on $X$ generates a topology $\tau_{p_{b}}$ on $X$, which has a subbase of the family of open $p_{b}$-balls $\left\{B_{p_{b}}(x, \varepsilon): x \in X, \varepsilon>0\right\}$, where $B_{p_{b}}(x, \varepsilon)=\left\{y \in X: p_{b}(x, y)<\right.$ $\left.p_{b}(x, x)+\varepsilon\right\}$, for all $x \in X$ and $\varepsilon>0$. The topology space $\left(X, p_{b}\right)$ is $T_{0}$, but does not need to be $T_{1}$. The topology $\tau_{p_{b}}$ on $X$ is called a $p_{b}$-metric topology.

Definition 1.4 ([17]) A sequence $\left\{x_{n}\right\}$ in a partial $b$-metric space is said to be:

(1) $p_{b}$-convergent to a point $x \in X$ if $\lim _{n \rightarrow \infty} p_{b}\left(x, x_{n}\right)=p_{b}(x, x)$.

(2) a $p_{b}$-Cauchy sequence if $\lim _{n, m \rightarrow \infty} p_{b}\left(x_{n}, x_{m}\right)$ exists and is finite.

(3) A partial $b$-metric space $\left(X, p_{b}\right)$ is said to be $p_{b}$-complete if every $p_{b}$-Cauchy sequence $\left\{x_{n}\right\}$ in $X p_{b}$-converges to a point $x \in X$ such that $\lim _{n \rightarrow \infty} p_{b}\left(x, x_{n}\right)=p_{b}(x, x)$.

It should be noted that the limit of a convergent sequence in a partial $b$-metric space may not be unique (see [16, Example 2]).

In [17], using Definition 1.3, Mustafa et al. proved the fact if $p_{b}$ is a partial $b$-metric on $X$, then the function $p_{b}^{s}: X \times X \rightarrow R^{+}$given by $p_{b}^{s}(x, y)=2 p_{b}(x, y)-p_{b}(x, x)-p_{b}(y, y)$ defines a $b$-metric on $X$. Using Definition 1.3, Mustafa et al. also obtained the following lemma which is the key to the proof of our theorems.

Lemma 1.1 ([17]) Let $\left(X, p_{b}\right)$ be a partial b-metric space. Then:

(1) A sequence $\left\{x_{n}\right\}$ in $X$ is a $p_{b}$-Cauchy sequence in $\left(X, p_{b}\right)$ if and only if it is a $b$-Cauchy sequence in $b$-metric space $\left(X, p_{b}^{s}\right)$.

(2) A partial b-metric space $\left(X, p_{b}\right)$ is $p_{b}$-complete if and only if the $b$-metric space $\left(X, p_{b}^{s}\right)$ is $b$-complete. Moreover, $\lim _{n \rightarrow \infty} p_{b}^{s}\left(x, x_{n}\right)=0$ if and only if $\lim _{n, m \rightarrow \infty} p_{b}\left(x_{n}, x_{m}\right)=\lim _{n \rightarrow \infty} p_{b}\left(x, x_{n}\right)=p_{b}(x, x)$.

It should be noted that in general a partial $b$-metric function $p_{b}(x, y)$ for $\lambda>1$ is not jointly continuous for all variables. The following example illustrates this fact.

Example 1.2 Let $X=N \cup\{\infty\}$, and let $p_{b}: X \times X \rightarrow R^{+}$be defined by

$$
p_{b}(m, n)= \begin{cases}0, & \text { if } m=n, \\ 6, & \text { if one of } m, n \text { is even and the other is even }(m \neq n) \text { or } \infty \\ \left|\frac{1}{m}-\frac{1}{n}\right|, & \text { if one of } m, n \text { is odd and the other is odd or } \infty \\ 3, & \text { otherwise }\end{cases}
$$

Then considering all possible cases, it can be checked that, for all $m, n, p \in X$, we have

$$
\begin{aligned}
p_{b}(m, p) & \leq 2\left[p_{b}(m, n)+p_{b}(n, p)\right] \\
& =2\left[p_{b}(m, n)+p_{b}(n, p)-p_{b}(n, n)\right]+\frac{1-2}{2}\left[p_{b}(m, m)+p_{b}(p, p)\right] .
\end{aligned}
$$


Thus, $\left(X, p_{b}\right)$ is a partial $b$-metric space (with $\lambda=2$ ). Let $x_{n}=2 n+1$ for each $n \in N$. Then $p_{b}(2 n+1, \infty)=\frac{1}{2 n+1} \rightarrow 0$ as $n \rightarrow \infty$, that is, $x_{n} \rightarrow \infty$, but $p_{b}\left(x_{n}, 2\right)=3 \nrightarrow 6=p_{b}(\infty, 2)$.

Since in general a partial $b$-metric is not continuous, we need the following simple lemma about the $p_{b}$-convergent sequences in the proof of our results.

Lemma 1.2 ([17]) Let $\left(X, p_{b}\right)$ be a partial b-metric space with the coefficient $\lambda \geq 1$ and suppose that $\left\{x_{n}\right\}$ and $\left\{y_{n}\right\}$ are $p_{b}$-convergent to $x$ and $y$, respectively. Then we have

$$
\begin{aligned}
\frac{p_{b}(x, y)}{\lambda^{2}}-\frac{p_{b}(x, x)}{\lambda}-p_{b}(y, y) & \leq \liminf _{n \rightarrow \infty} p_{b}\left(x_{n}, y_{n}\right) \leq \limsup _{n \rightarrow \infty} p_{b}\left(x_{n}, y_{n}\right) \\
& \leq \lambda p_{b}(x, x)+\lambda^{2} p_{b}(y, y)+\lambda^{2} p_{b}(x, y) .
\end{aligned}
$$

In particular, if $p_{b}(x, y)=0$, then we have $\lim _{n \rightarrow \infty} p_{b}\left(x_{n}, y_{n}\right)=0$. Moreover, for each $z \in X$, we have

$$
\frac{p_{b}(x, z)}{\lambda}-p_{b}(x, x) \leq \liminf _{n \rightarrow \infty} p_{b}\left(x_{n}, z\right) \leq \limsup _{n \rightarrow \infty} p_{b}\left(x_{n}, z\right) \leq \lambda p_{b}(x, z)+\lambda p_{b}(x, x) .
$$

In particular, if $p_{b}(x, x)=0$, then we have

$$
\frac{p_{b}(x, z)}{\lambda} \leq \liminf _{n \rightarrow \infty} p_{b}\left(x_{n}, z\right) \leq \limsup _{n \rightarrow \infty} p_{b}\left(x_{n}, z\right) \leq \lambda p_{b}(x, z) .
$$

Jungck [18] introduced the concept of weakly compatible mappings as follows.

Definition 1.5 ([18]) Let $X$ be a nonempty set, $A$ and $T: X \rightarrow X$ be two self-maps. $A$ and $T$ are said to be weakly compatible (or coincidentally commuting) if they commute at their coincidence points, i.e., if $A z=T z$ for some $z \in X$, then $A T z=T A z$.

It is worth mentioning that most of the preceding references concerned with fixed point results of contractions in partial metric spaces and $b$-metric spaces, but we rarely see fixed point results of expansions in such two types of spaces. Recently, in [19], Karapınar et al. considered a generalized expansive mapping and proved the fixed point theorem in metric spaces. Nashine et al. [20] introduced $\psi_{S}$-contractive mappings and proved some fixed point theorems in ordered metric spaces. Here, we recall the relevant definition.

Definition 1.6 ([20]) Let $(X, d, \leq)$ be an ordered metric space, and let $S, T: X \rightarrow X$. The mappings $S, T$ are said to be $\psi_{S}$-contractive if

$$
d(S x, T y) \leq \psi(d(x, y), d(x, S x), d(y, T y), d(x, T y), d(y, S x)),
$$

for all $x \geq y$, where $\psi: R^{+5} \rightarrow R^{+}$is a strictly increasing and continuous function in each coordinate, and for all $t \in R^{+} \backslash\{0\}, \psi(t, t, t, 0,2 t)<t, \psi(t, t, t, 2 t, 0)<t, \psi(0,0, t, t, 0)<t$, $\psi(0, t, 0,0, t)<t$ and $\psi(t, 0,0, t, t)<t$.

Inspired by the notions of $\psi_{S}$-contractive mappings of [20], we first introduce the concepts of generalized $(\psi, f)_{\lambda}$-expansive mappings and generalized $(\phi, g, h)_{\lambda}$-weakly expansive mappings. Then we establish some common fixed point theorems for these classes of 
mappings in complete partial $b$-metric spaces. The obtained results generalize and extend the main results of [15-23]. We also provide some examples to show the generality of our results. Finally, an application is given to illustrate the usability of the obtained results.

\section{Main results}

The study of expansive mappings is a very interesting research area in fixed point theory (see [19, 21-23]). In this section, inspired by the notion of $\psi_{S}$-contractive mappings of [20], we first introduce the notions of generalized $(\psi, f)_{\lambda}$-expansive mappings and generalized $(\phi, g, h)_{\lambda}$-weakly expansive mappings in partial $b$-metric spaces.

For convenience, we denote by $\Psi$ the class of functions $\psi: R^{+5} \rightarrow R^{+}$satisfying the following conditions:

(i) $\psi$ is a nondecreasing and continuous function in each coordinate;

(ii) for $t_{i} \in R^{+}, i=1,2, \ldots, 5, \psi\left(t_{1}, t_{2}, t_{3}, t_{4}, t_{5}\right)>\min \left\{t_{1}, \frac{t_{2}+t_{3}}{2}\right\}$, where $\min \left\{t_{1}, \frac{t_{2}+t_{3}}{2}\right\}>0$;

(iii) $\psi(0,0,0,0,0)=0$ and $\psi\left(t_{1}, t_{2}, t_{3}, t_{4}, t_{5}\right)>\min \left\{t_{1}, t_{5}\right\}$, where $\min \left\{t_{1}, t_{5}\right\}>0$.

The following are some easy examples of functions from class $\Psi$ :

$$
\begin{aligned}
& \psi\left(t_{1}, t_{2}, t_{3}, t_{4}, t_{5}\right)=a t_{1}, \text { for } a>1 ; \\
& \psi\left(t_{1}, t_{2}, t_{3}, t_{4}, t_{5}\right)=\max \left\{a \min \left\{t_{1}, \frac{t_{2}+t_{3}}{2}\right\}, c \min \left\{t_{1}, t_{5}\right\}\right\}, \text { for } a, c>1 ; \\
& \psi\left(t_{1}, t_{2}, t_{3}, t_{4}, t_{5}\right)=\max \left\{a t_{1}+b \frac{t_{2}+t_{3}}{2}, c \min \left\{t_{1}, t_{5}\right\}\right\}, \text { for } a, b \geq 0, a+b>1 \text {, and } c>1 ; \\
& \psi\left(t_{1}, t_{2}, t_{3}, t_{4}, t_{5}\right)=\max \left\{a t_{1}+b \frac{t_{2}+t_{3}}{2}, c t_{1}+d t_{5}\right\}, \text { for } a, b, c, d \geq 0, a+b>1, \text { and } c+d>1 ; \\
& \psi\left(t_{1}, t_{2}, t_{3}, t_{4}, t_{5}\right)=\max \left\{\min \left\{t_{1}, \frac{t_{2}+t_{3}}{2}\right\}, \min \left\{t_{1}, t_{5}\right\}\right\}+\phi\left(\max \left\{\min \left\{t_{1}, \frac{t_{2}+t_{3}}{2}\right\}, \min \left\{t_{1}, t_{5}\right\}\right\}\right), \\
& \text { where } \phi: R^{+} \rightarrow R^{+} \text {is a nondecreasing and continuous function, and } \phi(s)=0 \text { if and } \\
& \text { only if } s=0 .
\end{aligned}
$$

Definition 2.1 Let $\left(X, p_{b}\right)$ be a partial $b$-metric space with the coefficient $\lambda \geq 1, A, S$, and $T: X \rightarrow X$ be three mappings. Then $A, S$, and $T$ are said to be generalized $(\psi, f)_{\lambda}$ expansive mappings if

$$
f\left(\frac{p_{b}(S x, T y)}{\lambda^{2}}\right) \geq \psi\left(p_{b}(A x, A y), p_{b}(A x, S x), p_{b}(A y, T y), \frac{p_{b}(A x, T y)}{\lambda}, \frac{p_{b}(A y, S x)}{\lambda}\right),
$$

for all $x, y \in X$, where $\psi \in \Psi, f:[0, \infty) \rightarrow[0, \infty)$ is a nondecreasing and continuous function, $f(0)=0$, and for all $t>0, \psi\left(t_{1}, t_{2}, t_{3}, t_{4}, t_{5}\right)>f(t)$, where $\min \left\{t_{1}, \frac{t_{2}+t_{3}}{2}\right\}=t$ or $\min \left\{t_{1}, t_{5}\right\}=t$.

Definition 2.2 Let $\left(X, p_{b}\right)$ be a partial $b$-metric space with the coefficient $\lambda \geq 1, A, S$, and $T: X \rightarrow X$ be three mappings. Then $A, S$, and $T$ are said to be generalized $(\phi, g, h)_{\lambda}$-weakly expansive mappings if

$$
g\left(\frac{p_{b}(S x, T y)}{\lambda^{2}}\right) \geq h\left(M_{\lambda}(A x, A y)\right)+\phi\left(M_{\lambda}(A x, A y)\right)
$$

for all $x, y \in X$, where $M_{\lambda}(A x, A y)=\max \left\{\min \left\{p_{b}(A x, A y), \frac{p_{b}(A x, S x)+p_{b}(A y, T y)}{2}\right\}, \min \left\{p_{b}(A x, A y)\right.\right.$, $\left.\left.\frac{p_{b}(A y, S x)}{\lambda}\right\}\right\}, \phi, g, h: R^{+} \rightarrow R^{+}$are continuous and nondecreasing functions, $g(0)=h(0)=0$, $\phi(s)=0$ if and only if $s=0$, and for all $t>0, h(t)+\phi(t)>g(t)$.

It is easy to acquire the following example of generalized $(\psi, f)_{\lambda}$-expansive mappings or generalized $(\phi, g, h)_{\lambda}$-weakly expansive mappings. 
Example 2.1 Let $X=R^{+2}$ be endowed with the partial $b$-metric $p_{b}: X \times X \rightarrow R^{+}$given by

$$
p_{b}(u, v)= \begin{cases}0, & \text { if } x_{1}=x_{2} \text { and } y_{1}=y_{2}, \\ \left(x_{1}+x_{2}\right)^{2}, & \text { if } x_{1} \neq x_{2} \text { and } y_{1}=y_{2}, \\ \left(y_{1}+y_{2}\right)^{2}, & \text { if } x_{1}=x_{2} \text { and } y_{1} \neq y_{2} \\ \left(x_{1}+x_{2}\right)^{2}+\left(y_{1}+y_{2}\right)^{2}, & \text { otherwise }\end{cases}
$$

for $u=\left(x_{1}, y_{1}\right), v=\left(x_{2}, y_{2}\right) \in X$, where $\lambda=2$. Let $\psi: R^{+5} \rightarrow R^{+}$and $f: R^{+} \rightarrow R^{+}$be given by

$$
\psi\left(t_{1}, t_{2}, t_{3}, t_{4}, t_{5}\right)=\max \left\{2 \min \left\{t_{1}, \frac{t_{2}+t_{3}}{2}\right\}, 2 \min \left\{t_{1}, t_{5}\right\}\right\}, \quad f(t)=t
$$

for all $t_{1}, t_{2}, \ldots, t_{5}, t \in R^{+}$, and $A, S, T: X \rightarrow X$ be given by

$$
\begin{aligned}
& S(x, y)=\left(3 x, e^{2 y}-1+y\right), \quad T(x, y)=\left(3 x, e^{2 y}-1+y\right), \\
& A(x, y)=\left(\frac{3}{4} x, \frac{3}{4} y\right), \quad \text { for all }(x, y) \in X .
\end{aligned}
$$

Then $A, S$, and $T$ are generalized $(\psi, f)_{\lambda}$-expansive mappings. In fact, if $\phi, g, h: R^{+} \rightarrow R^{+}$ are defined by

$$
g(t)=\frac{t}{2}, \quad h(t)=\eta t, \quad \phi(t)=(1-\eta) t
$$

for all $t \in R^{+}$, where $0<\eta<1$. Then $A, S$, and $T$ are also generalized $(\phi, g, h)_{\lambda}$-weakly expansive mappings.

Now, we first prove some fixed point results for generalized $(\psi, f)_{\lambda}$-expansive mappings in $p_{b}$-complete partial $b$-metric spaces.

Theorem 2.1 Let $\left(X, p_{b}\right)$ be a $p_{b}$-complete partial b-metric space, $A, S$, and $T: X \rightarrow X$ be three mappings satisfying the generalized $(\psi, f)_{\lambda}$-expansive condition (2.1). Suppose that the following conditions are satisfied:

(i) $A(X) \subset S(X), A(X) \subset T(X)$, and $A(X)$ is a closed subset of $\left(X, p_{b}^{s}\right)$;

(ii) $A$ is an injective and $A$ and $T$ are weakly compatible.

Then $A, S$, and $T$ have a unique common fixed point in $X$.

Proof Let $x_{0} \in X$ be an arbitrary point in $X$. Since $A(X) \subset S(X)$, there exists an $x_{1} \in X$ such that $A x_{0}=S x_{1}$. Since $A(X) \subset T(X)$, there exists an $x_{2} \in X$ such that $A x_{1}=T x_{2}$. Continuing this process, we can construct a sequence $\left\{A x_{n}\right\}$ in $X$ such that

$$
A x_{2 n}=S x_{2 n+1}, \quad A x_{2 n+1}=T x_{2 n+2}, \quad \text { for } n=0,1,2, \ldots
$$

We will complete the proof in three steps.

Step 1 . We prove that

$$
\lim _{n \rightarrow \infty} p_{b}\left(A x_{n}, A x_{n+1}\right)=0 .
$$


Suppose that $p_{b}\left(A x_{n}, A x_{n+1}\right)=0$ for some $n=n_{0}$. In the case that $n_{0}=2 k, p_{b}\left(A x_{2 k}\right.$, $\left.A x_{2 k+1}\right)=0$ gives $p_{b}\left(A x_{2 k+1}, A x_{2 k+2}\right)=0$. Indeed, by (2.1), we have

$$
\begin{aligned}
0= & f\left(\frac{p_{b}\left(A x_{2 k}, A x_{2 k+1}\right)}{\lambda^{2}}\right)=f\left(\frac{p_{b}\left(S x_{2 k+1}, T x_{2 k+2}\right)}{\lambda^{2}}\right) \\
\geq & \psi\left(p_{b}\left(A x_{2 k+1}, A x_{2 k+2}\right), p_{b}\left(A x_{2 k+1}, S x_{2 k+1}\right),\right. \\
& \left.p_{b}\left(A x_{2 k+2}, T x_{2 k+2}\right), \frac{p_{b}\left(A x_{2 k+1}, T x_{2 k+2}\right)}{\lambda}, \frac{p_{b}\left(A x_{2 k+2}, S x_{2 k+1}\right)}{\lambda}\right) \\
= & \psi\left(p_{b}\left(A x_{2 k+1}, A x_{2 k+2}\right), 0, p_{b}\left(A x_{2 k+1}, A x_{2 k+2}\right), 0, \frac{p_{b}\left(A x_{2 k+2}, A x_{2 k}\right)}{\lambda}\right),
\end{aligned}
$$

which implies that $\frac{p_{b}\left(A x_{2 k+1}, A x_{2 k+2}\right)}{2}=0$, that is, $p_{b}\left(A x_{2 k+1}, A x_{2 k+2}\right)=0$. Similarly, if $n_{0}=$ $2 k+1$, then $p_{b}\left(A x_{2 k+2}, A x_{2 k+3}\right)=0$. Consequently, $p_{b}\left(A x_{n}, A x_{n+1}\right) \equiv 0$ for $n \geq n_{0}$. Hence, $\lim _{n \rightarrow \infty} p_{b}\left(A x_{n}, A x_{n+1}\right)=0$.

Now, suppose that $p_{b}\left(A x_{n}, A x_{n+1}\right)>0$, for each $n$. By (2.1), we have

$$
\begin{aligned}
f\left(\frac{p_{b}\left(A x_{2 n}, A x_{2 n+1}\right)}{\lambda^{2}}\right)= & f\left(p_{b}\left(S x_{2 n+1}, T x_{2 n+2}\right)\right) \\
\geq & \psi\left(p_{b}\left(A x_{2 n+1}, A x_{2 n+2}\right), p_{b}\left(A x_{2 n+1}, S x_{2 n+1}\right),\right. \\
& \left.p_{b}\left(A x_{2 n+2}, T x_{2 n+2}\right), \frac{p_{b}\left(A x_{2 n+1}, T x_{2 n+2}\right)}{\lambda}, \frac{p_{b}\left(A x_{2 n+2}, S x_{2 n+1}\right)}{\lambda}\right) \\
= & \psi\left(p_{b}\left(A x_{2 n+1}, A x_{2 n+2}\right), p_{b}\left(A x_{2 n}, A x_{2 n+1}\right), p_{b}\left(A x_{2 n+1}, A x_{2 n+2}\right),\right. \\
& \left.\frac{p_{b}\left(A x_{2 n+1}, A x_{2 n+1}\right)}{\lambda}, \frac{p_{b}\left(A x_{2 n+2}, A x_{2 n}\right)}{\lambda}\right) .
\end{aligned}
$$

If $\min \left\{p_{b}\left(A x_{2 n+1}, A x_{2 n+2}\right), \frac{p_{b}\left(A x_{2 n}, A x_{2 n+1}\right)+p_{b}\left(A x_{2 n+1}, A x_{2 n+2}\right)}{2}\right\}=\frac{p_{b}\left(A x_{2 n}, A x_{2 n+1}\right)+p_{b}\left(A x_{2 n+1}, A x_{2 n+2}\right)}{2}>0$, then we have $p_{b}\left(A x_{2 n}, A x_{2 n+1}\right) \leq p_{b}\left(A x_{2 n+1}, A x_{2 n+2}\right)$. It follows from (2.4) and the properties of $\psi$ and $f$ that

$$
\begin{aligned}
f\left(p_{b}\left(A x_{2 n}, A x_{2 n+1}\right)\right) \geq & f\left(\frac{p_{b}\left(A x_{2 n}, A x_{2 n+1}\right)}{\lambda^{2}}\right) \geq \psi\left(p_{b}\left(A x_{2 n+1}, A x_{2 n+2}\right), p_{b}\left(A x_{2 n}, A x_{2 n+1}\right),\right. \\
& \left.p_{b}\left(A x_{2 n+1}, A x_{2 n+2}\right), \frac{p_{b}\left(A x_{2 n+1}, A x_{2 n+1}\right)}{\lambda}, \frac{p_{b}\left(A x_{2 n+2}, A x_{2 n}\right)}{\lambda}\right) \\
> & f\left(\frac{p_{b}\left(A x_{2 n}, A x_{2 n+1}\right)+p_{b}\left(A x_{2 n+1}, A x_{2 n+2}\right)}{2}\right) .
\end{aligned}
$$

Since $f$ is nondecreasing, we get $p_{b}\left(A x_{2 n}, A x_{2 n+1}\right)>p_{b}\left(A x_{2 n+1}, A x_{2 n+2}\right)$, which is a contradiction. Thus,

$$
f\left(p_{b}\left(A x_{2 n}, A x_{2 n+1}\right)\right) \geq f\left(\frac{p_{b}\left(A x_{2 n}, A x_{2 n+1}\right)}{\lambda^{2}}\right)>f\left(p_{b}\left(A x_{2 n+1}, A x_{2 n+2}\right)\right) .
$$

Hence, we deduce that, for each $n \in N, p_{b}\left(A x_{2 n+1}, A x_{2 n+2}\right)<p_{b}\left(A x_{2 n}, A x_{2 n+1}\right)$. Similarly, we can prove that $p_{b}\left(A x_{2 n}, A x_{2 n+1}\right)<p_{b}\left(A x_{2 n-1}, A x_{2 n}\right)$, for all $n \geq 1$. Therefore, $\left\{p_{b}\left(A x_{n}, A x_{n+1}\right)\right\}$ 
is a decreasing sequence of nonnegative real numbers. So, there exists $r \geq 0$ such that $\lim _{n \rightarrow \infty} p_{b}\left(A x_{n}, A x_{n+1}\right)=r$.

From Definition 1.3 $\left(\mathrm{p}_{b 4}^{\prime}\right)$, we have

$$
\begin{aligned}
p_{b}\left(A x_{n}, A x_{n+2}\right) & \leq p_{b}\left(A x_{n}, A x_{n+2}\right)+p_{b}\left(A x_{n+1}, A x_{n+1}\right) \\
& \leq \lambda p_{b}\left(A x_{n}, A x_{n+1}\right)+\lambda p_{b}\left(A x_{n+1}, A x_{n+2}\right) .
\end{aligned}
$$

It follows from (2.5) that $\left\{p_{b}\left(A x_{n}, A x_{n+2}\right)\right\}$ and $\left\{p_{b}\left(A x_{n+1}, A x_{n+1}\right)\right\}$ are two bounded sequences. Hence, the sequence $\left\{p_{b}\left(A x_{n}, A x_{n+2}\right)\right\}$ has a subsequence $\left\{p_{b}\left(A x_{n_{k}}, A x_{n_{k}+2}\right)\right\}$ which converges to a real number $\alpha \leq 2 \lambda r$, and the sequence $\left\{p_{b}\left(A x_{n_{k}+1}, A x_{n_{k}+1}\right)\right\}$ has a subsequence $\left\{p_{b}\left(A x_{n_{k(i)}+1}, A x_{n_{k(i)}+1}\right)\right\}$ which converges to a real number $\beta \leq 2 \lambda r$. By (2.4), we have

$$
\begin{aligned}
f\left(\frac{p_{b}\left(A x_{2 n_{k(i)},}, A x_{2 n_{k(i)}+1}\right)}{\lambda^{2}}\right)= & f\left(p_{b}\left(S x_{2 n_{k(i)}+1}, T x_{2 n_{k(i)}+2}\right)\right) \\
\geq & \psi\left(p_{b}\left(A x_{2 n_{k(i)}+1}, A x_{2 n_{k(i)}+2}\right), p_{b}\left(A x_{2 n_{k(i)}}, A x_{2 n_{k(i)}+1}\right),\right. \\
& p_{b}\left(A x_{2 n_{k(i)}+1}, A x_{2 n_{k(i)}+2}\right), \\
& \left.\frac{p_{b}\left(A x_{2 n_{k(i)}+1}, A x_{2 n_{k(i)}+1}\right)}{\lambda}, \frac{p_{b}\left(A x_{2 n_{k(i)}+2}, A x_{2 n_{k(i)}}\right)}{\lambda}\right) .
\end{aligned}
$$

Letting $n_{k(i)} \rightarrow \infty$ in the above inequality, by the properties of $\psi$ and $f$, we have $f(r) \geq$ $f\left(\frac{r}{\lambda^{2}}\right) \geq \psi\left(r, r, r, \frac{\beta}{\lambda}, \frac{\alpha}{\lambda}\right)$, which implies that $r=0$. Hence, $\lim _{n \rightarrow \infty} p\left(A x_{n}, A x_{n+1}\right)=0$.

Step 2. We show that $\left\{A x_{n}\right\}$ is a $p_{b}$-Cauchy sequence.

Indeed, we first prove that $\lim _{m, n \rightarrow \infty} p_{b}\left(A x_{n}, A x_{m}\right)=0$. Because of (2.3), it is sufficient to show that $\lim _{m, n \rightarrow \infty} p_{b}\left(A x_{2 n}, A x_{2 m}\right)=0$. Suppose on the contrary, then there exists $\varepsilon>0$ for which we can find two subsequences $\left\{A x_{2 n(k)}\right\}$ and $\left\{A x_{2 m(k)}\right\}$ of $\left\{A x_{2 n}\right\}$ such that $m(k)$ is the smallest index for which

$$
m(k)>n(k)>k, \quad p_{b}\left(A x_{2 m(k)}, A_{2 n(k)}\right) \geq \varepsilon, \quad \text { for every } k .
$$

This means that

$$
p_{b}\left(A x_{2 m(k)-2}, A_{2 n(k)}\right)<\varepsilon .
$$

From (2.6), using the triangular inequality, we can see that

$$
0<\varepsilon \leq p_{b}\left(A x_{2 m(k)}, A x_{2 n(k)}\right) \leq \lambda p_{b}\left(A x_{2 m(k)}, A x_{2 n(k)+1}\right)+\lambda p_{b}\left(A x_{2 n(k)+1}, A x_{2 n(k)}\right)
$$

and

$$
\begin{aligned}
0 & <\varepsilon \leq p_{b}\left(A x_{2 m(k)}, A x_{2 n(k)}\right) \leq \lambda p_{b}\left(A x_{2 m(k)}, A x_{2 m(k)-1}\right)+\lambda p_{b}\left(A x_{2 m(k)-1}, A x_{2 n(k)}\right) \\
& \leq \lambda p_{b}\left(A x_{2 m(k)}, A x_{2 m(k)-1}\right)+\lambda^{2} p_{b}\left(A x_{2 m(k)-1}, A x_{2 n(k)+1}\right)+\lambda^{2} p_{b}\left(A x_{2 n(k)+1}, A x_{2 n(k)}\right) .
\end{aligned}
$$


By means of (2.3), taking the lower limit as $k \rightarrow \infty$ in the above inequality, we get

$$
\begin{aligned}
& \varepsilon \leq \liminf _{k \rightarrow \infty} p_{b}\left(A x_{2 m(k)}, A x_{2 n(k)}\right) \leq \limsup _{k \rightarrow \infty} p_{b}\left(A x_{2 m(k)}, A x_{2 n(k)}\right) . \\
& \frac{\varepsilon}{\lambda} \leq \liminf _{k \rightarrow \infty} p_{b}\left(A x_{2 m(k)}, A x_{2 n(k)+1}\right) \leq \limsup _{k \rightarrow \infty} p_{b}\left(A x_{2 m(k)}, A x_{2 n(k)+1}\right), \\
& \frac{\varepsilon}{\lambda^{2}} \leq \liminf _{k \rightarrow \infty} p_{b}\left(A x_{2 m(k)-1}, A x_{2 n(k)+1}\right) \leq \limsup _{k \rightarrow \infty} p_{b}\left(A x_{2 m(k)-1}, A x_{2 n(k)+1}\right) .
\end{aligned}
$$

On the other hand, we have

$$
p_{b}\left(A x_{2 n(k)}, A x_{2 m(k)-1}\right) \leq \lambda p_{b}\left(A x_{2 n(k)}, A x_{2 m(k)-2}\right)+\lambda p_{b}\left(A x_{2 m(k)-2}, A x_{2 m(k)-1}\right) .
$$

With the help of (2.3) and (2.7) and taking the upper limit as $k \rightarrow \infty$ in the above inequality, it is not difficult to see that

$$
\limsup _{k \rightarrow \infty} p_{b}\left(A x_{2 n(k)}, A x_{2 m(k)-1}\right) \leq \lambda \varepsilon
$$

From (2.1), we have

$$
\begin{aligned}
f( & \left.\frac{p_{b}\left(A x_{2 n(k)}, A x_{2 m(k)-1}\right)}{\lambda^{2}}\right) \\
= & f\left(\frac{p_{b}\left(S x_{2 n(k)+1}, T x_{2 m(k)}\right)}{\lambda^{2}}\right) \\
\geq & \psi\left(p_{b}\left(A x_{2 n(k)+1}, A x_{2 m(k)}\right), p_{b}\left(A x_{2 n(k)+1}, S x_{2 n(k)+1}\right),\right. \\
& \left.p_{b}\left(A x_{2 m(k)}, T x_{2 m(k)}\right), \frac{p_{b}\left(A x_{2 n(k)+1}, T x_{2 m(k)}\right)}{\lambda}, \frac{p_{b}\left(A x_{2 m(k)}, S x_{2 n(k)+1}\right)}{\lambda}\right) \\
= & \psi\left(p_{b}\left(A x_{2 n(k)+1}, A x_{2 m(k)}\right), p_{b}\left(A x_{2 n(k)+1}, A x_{2 n(k)}\right),\right. \\
& \left.p_{b}\left(A x_{2 m(k)}, A x_{2 m(k)-1}\right), \frac{p_{b}\left(A x_{2 n(k)+1}, A x_{2 m(k)-1}\right)}{\lambda}, \frac{p_{b}\left(A x_{2 m(k)}, A x_{2 n(k)}\right)}{\lambda}\right) .
\end{aligned}
$$

Now, taking upper limit as $k \rightarrow \infty$ in the above inequality, the properties of $\psi, f$, and (2.8)-(2.11) guarantee that

$$
\begin{aligned}
f\left(\frac{\varepsilon}{\lambda}\right) \geq & f\left(\frac{\limsup _{k \rightarrow \infty} p_{b}\left(A x_{2 n(k)}, A x_{2 m(k)-1}\right)}{\lambda^{2}}\right) \\
= & f\left(\frac{\limsup _{k \rightarrow \infty} p_{b}\left(S x_{2 n(k)+1}, T x_{2 m(k)}\right)}{\lambda^{2}}\right) \\
\geq & \psi\left(\liminf _{k \rightarrow \infty} p_{b}\left(A x_{2 n(k)+1}, A x_{2 m(k)}\right), \liminf _{k \rightarrow \infty} p_{b}\left(A x_{2 n(k)+1}, A x_{2 n(k)}\right),\right. \\
& \liminf _{k \rightarrow \infty} p_{b}\left(A x_{2 m(k)}, A x_{2 m(k)-1}\right), \\
& \left.\frac{\liminf _{k \rightarrow \infty} p_{b}\left(A x_{2 n(k)+1}, A x_{2 m(k)-1}\right)}{\lambda}, \frac{\liminf _{k \rightarrow \infty} p_{b}\left(A x_{2 m(k)}, A x_{2 n(k)}\right)}{\lambda}\right),
\end{aligned}
$$


which implies that $f\left(\frac{\varepsilon}{\lambda}\right) \geq \psi\left(\frac{\varepsilon}{\lambda}, 0,0, \frac{\varepsilon}{\lambda^{3}}, \frac{\varepsilon}{\lambda}\right)$. Thus, $\varepsilon=0$, a contradiction. Hence, $\lim _{m, n \rightarrow \infty} p_{b}\left(A x_{n}, A x_{m}\right)=0$, that is, $\left\{A x_{n}\right\}$ is a $p_{b}$-Cauchy sequence.

Step 3. We will show that $A, S$, and $T$ have a unique common fixed point.

Since $\left\{A x_{n}\right\}$ is a $p_{b}$-Cauchy sequence in $\left(X, p_{b}\right)$, and thus it is also $b$-Cauchy sequence in the $b$-metric space $\left(X, p_{b}^{s}\right)$ by Lemma 1.1. Since $\left(X, p_{b}\right)$ is $p_{b}$-complete, from Lemma 1.1, $\left(X, p_{b}^{s}\right)$ is also $b$-complete, so the sequence $\left\{A x_{n}\right\}$ is $b$-convergent in the $b$-metric space $\left(X, p_{b}^{s}\right)$. Therefore, there exists $x^{*} \in X$ such that $\lim _{n \rightarrow \infty} p_{b}^{s}\left(A x_{n}, x^{*}\right)=0$. Then $\lim _{m, n \rightarrow \infty} p_{b}\left(A x_{n}, A x_{m}\right)=\lim _{n \rightarrow \infty} p_{b}\left(A x_{n}, x^{*}\right)=p_{b}\left(x^{*}, x^{*}\right)=0$.

Since $A(X)$ is a closed set of $\left(X, p_{b}^{s}\right), A(X) \subset T(X)$, and $\lim _{n \rightarrow \infty} p_{b}^{s}\left(A x_{n}, x^{*}\right)=0$, we get $x^{*} \in A(X) \subset T(X)$. Hence, there exists $z_{1} \in X$ such that $T z_{1}=x^{*}$. This together with (2.1) ensures that

$$
\begin{aligned}
f\left(\frac{p_{b}\left(A x_{2 n}, T z_{1}\right)}{\lambda^{2}}\right)= & f\left(\frac{p_{b}\left(S x_{2 n+1}, T z_{1}\right)}{\lambda^{2}}\right) \\
\geq & \psi\left(p_{b}\left(A x_{2 n+1}, A z_{1}\right), p_{b}\left(A x_{2 n+1}, S x_{2 n+1}\right),\right. \\
& \left.p_{b}\left(A z_{1}, T z_{1}\right), \frac{p_{b}\left(A x_{2 n+1}, T z_{1}\right)}{\lambda}, \frac{p_{b}\left(A z_{1}, S x_{2 n+1}\right)}{\lambda}\right) \\
= & \psi\left(p_{b}\left(A x_{2 n+1}, A z_{1}\right), p_{b}\left(A x_{2 n+1}, A x_{2 n}\right),\right. \\
& \left.p_{b}\left(A z_{1}, T z_{1}\right), \frac{p_{b}\left(A x_{2 n+1}, T z_{1}\right)}{\lambda}, \frac{p_{b}\left(A z_{1}, A x_{2 n}\right)}{\lambda}\right) .
\end{aligned}
$$

Since $\lim _{n \rightarrow \infty} p_{b}\left(A x_{n}, x^{*}\right)=p_{b}\left(x^{*}, x^{*}\right)=0$ and $T z_{1}=x^{*}$, we can find by Lemma 1.1 that

$$
\begin{array}{ll}
\limsup _{n \rightarrow \infty} p_{b}\left(A x_{2 n}, T z_{1}\right)=0, & \liminf _{n \rightarrow \infty} p_{b}\left(A x_{2 n+1}, A z_{1}\right) \geq \frac{p_{b}\left(T z_{1}, A z_{1}\right)}{\lambda}, \\
\liminf _{n \rightarrow \infty} p_{b}\left(A x_{2 n+1}, T z_{1}\right)=0, & \liminf _{n \rightarrow \infty} p_{b}\left(A z_{1}, A x_{2 n}\right) \geq \frac{p_{b}\left(A z_{1}, T z_{1}\right)}{\lambda} .
\end{array}
$$

Taking the upper limit as $n \rightarrow \infty$ in (2.12), using the properties of $\psi$ and $f$, (2.13), and (2.14), it is clear that

$$
\begin{aligned}
0 & =f(0)=f\left(\limsup _{n \rightarrow \infty} p_{b}\left(A x_{2 n}, T z_{1}\right)\right) \\
& \geq \psi\left(\frac{p_{b}\left(A z_{1}, T z_{1}\right)}{\lambda}, 0, p_{b}\left(A z_{1}, T z_{1}\right), 0, \frac{p_{b}\left(A z_{1}, T z_{1}\right)}{\lambda^{2}}\right),
\end{aligned}
$$

which implies that $p_{b}\left(A z_{1}, T z_{1}\right)=0$. Hence, $A z_{1}=T z_{1}=x^{*}$. Similarly, since $x^{*} \in A(X) \subset$ $S(X)$, there exists $z_{2} \in X$ such that $S z_{2}=x^{*}$, we have $A z_{2}=S z_{2}=x^{*}$. Hence, $A z_{1}=A z_{2}=x^{*}$. Since $A$ is an injective, we get $z_{1}=z_{2}$.

Let $z=z_{1}=z_{2}$. Then $A z=S z=T z=x^{*}$. Since $A$ and $T$ are weakly compatible, it is obvious that $A x^{*}=A A z=A T z=T A z=T x^{*}$. By (2.1), we get

$$
\begin{aligned}
f\left(\frac{p_{b}\left(A z, A x^{*}\right)}{\lambda}\right) & \geq f\left(\frac{p_{b}\left(A z, A x^{*}\right)}{\lambda^{2}}\right)=f\left(\frac{p_{b}\left(S z, T x^{*}\right)}{\lambda^{2}}\right) \\
& \geq \psi\left(p_{b}\left(A z, A x^{*}\right), p_{b}(A z, S z), p_{b}\left(A x^{*}, T x^{*}\right)\right.
\end{aligned}
$$




$$
\begin{aligned}
& \left.\frac{p_{b}\left(A z, T x^{*}\right)}{\lambda}, \frac{p_{b}\left(A x^{*}, S z\right)}{\lambda}\right) \\
= & \psi\left(p_{b}\left(A z, A x^{*}\right), p_{b}(A z, A z), p_{b}\left(A x^{*}, A x^{*}\right),\right. \\
& \left.\frac{p_{b}\left(A z, A x^{*}\right)}{\lambda}, \frac{p_{b}\left(A x^{*}, A z\right)}{\lambda}\right)
\end{aligned}
$$

which implies that $p_{b}\left(A z, A x^{*}\right)=0$. Thus, $A z=A x^{*}$. Since $A$ is an injective, we get $z=x^{*}$. Hence, $A z=S z=T z=z$, that is, $z$ is a common fixed point of $A, S$, and $T$.

Now, we prove the uniqueness of common fixed points of $A, S$, and $T$. Suppose that $x^{*}, y^{*} \in X$ such that $A x^{*}=S x^{*}=T x^{*}=x^{*}$ and $A y^{*}=S y^{*}=T y^{*}=y^{*}$. By means of (2.1), we have

$$
\begin{aligned}
f\left(\frac{p_{b}\left(x^{*}, y^{*}\right)}{\lambda}\right) \geq & f\left(\frac{p_{b}\left(x^{*}, y^{*}\right)}{\lambda^{2}}\right)=f\left(\frac{p_{b}\left(S x^{*}, T y^{*}\right)}{\lambda^{2}}\right) \\
\geq & \psi\left(p_{b}\left(A x^{*}, A y^{*}\right), p_{b}\left(A x^{*}, S x^{*}\right), p_{b}\left(A y^{*}, T y^{*}\right),\right. \\
& \left.\frac{p_{b}\left(A x^{*}, T y^{*}\right)}{\lambda}, \frac{p_{b}\left(A y^{*}, S x^{*}\right)}{\lambda}\right) \\
= & \psi\left(p_{b}\left(x^{*}, y^{*}\right), p_{b}\left(x^{*}, x^{*}\right), p_{b}\left(x^{*}, y^{*}\right), \frac{p_{b}\left(x^{*}, y^{*}\right)}{\lambda}, \frac{p_{b}\left(y^{*}, x^{*}\right)}{\lambda}\right),
\end{aligned}
$$

which implies that $p_{b}\left(x^{*}, y^{*}\right)=0$. Hence, $x^{*}=y^{*}$. This completes the proof.

Remark 2.1 Let $I$ be the identity mappings on $X$. Taking $A=I, f(t)=t$, for all $t \in R^{+}$in Theorem 2.1, we have the following corollary, which extends and generalizes Theorem 2.1 in [19] and Theorem 2 in [20].

Corollary 2.1 Let $\left(X, p_{b}\right)$ be a $p_{b}$-complete partial b-metric space, $S$ and $T: X \rightarrow X$ be two bijective mappings. Suppose that

$$
\frac{p_{b}(S x, T y)}{\lambda^{2}} \geq \psi\left(p_{b}(x, y), p_{b}(x, S x), p_{b}(y, T y), \frac{p_{b}(x, T y)}{\lambda}, \frac{p_{b}(y, S x)}{\lambda}\right),
$$

for all $x, y \in X$, where $\psi \in \Psi$. Then $S$ and $T$ have a unique common fixed point in $X$.

Now, in order to support the usability of our results, we present the following example.

Example 2.2 Let $C[0,1]$ be the set of all real continuous functions defined on $[0,1]$ and $X=\{x \geq \theta: x \in C[0,1]\}$. Define a partial $b$-metric $p_{b}: X \times X \rightarrow R^{+}$by

$$
p_{b}(x, y)= \begin{cases}\max _{t \in[0,1]}|x(t)|^{2}, & \text { if } x=y, \\ \max _{t \in[0,1]}(x(t)+y(t))^{2}, & \text { otherwise. }\end{cases}
$$

It is easy to see that $\left(X, p_{b}\right)$ is a $p_{b}$-complete partial $b$-metric space with $\lambda=3$. Let $A, S, T$ : $X \rightarrow X$ be defined by

$$
(A x)(t)=\frac{2}{3} \int_{0}^{t} x(s) d s, \quad(S x)(t)=4 \int_{0}^{t} x(s) d s,
$$




$$
(T x)(t)=7 \int_{0}^{t} x(s) d s, \quad \text { for all } x \in X
$$

Then it is easy to show that all the conditions (i)-(ii) of Theorem 2.1 are satisfied. Define $\psi$ : $R^{+5} \rightarrow R^{+}$and $f: R^{+} \rightarrow R^{+}$by $\psi\left(t_{1}, t_{2}, t_{3}, t_{4}, t_{5}\right)=\max \left\{\frac{6}{5} \min \left\{t_{1}, \frac{t_{1}+t_{2}}{2}\right\}, \frac{6}{5} \min \left\{t_{1}, t_{5}\right\}\right\}, f(t)=t$.

Now, we consider following cases:

Case 1. If $x=y=\theta$, then $\frac{p_{b}(S x, T y)}{9}=0=\frac{6}{5} p_{b}(A x, A y)$.

Case 2. If $x=y \neq \theta$, then

$$
\begin{aligned}
\frac{p_{b}(S x, T y)}{9} & =\frac{\max _{t \in[0,1]}\left(4 \int_{0}^{t} x(s) d s+7 \int_{0}^{t} y(s) d s\right)^{2}}{9} \\
& =\frac{121}{9} \max _{t \in[0,1]}\left(\int_{0}^{t} x(s) d s\right)^{2} \geq \frac{8}{15} \max _{t \in[0,1]}\left(\int_{0}^{t} x(s) d s\right)^{2}=\frac{6}{5} p_{b}(A x, A y) .
\end{aligned}
$$

Case 3. If $x \neq y$ and $4 x=7 y$, then

$$
\begin{aligned}
\frac{p_{b}(S x, T y)}{9} & =\frac{16}{9} \max _{t \in[0,1]}\left(\int_{0}^{t} x(s) d s\right)^{2} \geq \frac{8}{15} \max _{t \in[0,1]}\left(\int_{0}^{t} x(s) d s+\frac{4}{7} \int_{0}^{t} x(s) d s\right)^{2} \\
& =\frac{6}{5} p_{b}(A x, A y) .
\end{aligned}
$$

Case 4 . If $x \neq y$ and $4 x \neq 7 y$, then

$$
\begin{aligned}
\frac{p_{b}(S x, T y)}{9} & =\frac{\max _{t \in[0,1]}\left(4 \int_{0}^{t} x(s) d s+7 \int_{0}^{t} y(s) d s\right)^{2}}{9} \\
& \geq \frac{16}{9} \max _{t \in[0,1]}\left(\int_{0}^{t} x(s) d s+\int_{0}^{t} y(s) d s\right)^{2} \\
& \geq \frac{8}{15} \max _{t \in[0,1]}\left(\int_{0}^{t} x(s) d s+\int_{0}^{t} y(s) d s\right)^{2}=\frac{6}{5} p_{b}(A x, A y) .
\end{aligned}
$$

That is,

$$
\begin{aligned}
\frac{p_{b}(S x, T y)}{\lambda^{2}} & \geq \frac{6}{5} p_{b}(A x, A y) \\
& \geq \psi\left(p_{b}(A x, A y), p_{b}(A x, S x), p_{b}(A y, T y), \frac{p_{b}(A x, T y)}{\lambda}, \frac{p_{b}(A y, S x)}{\lambda}\right),
\end{aligned}
$$

for all $x, y \in X$. Thus, all conditions of Theorem 2.1 are satisfied. Hence, $A, S$, and $T$ have a unique common fixed point $x=\theta$.

Now, we state and prove some fixed point results for generalized $(\phi, g, h)_{\lambda}$-weakly expansive mappings in partial $b$-metric spaces.

Theorem 2.2 Let $\left(X, p_{b}\right)$ be a $p_{b}$-complete partial b-metric space, $A, S$, and $T: X \rightarrow X$ be three mappings satisfying the generalized $(\phi, g, h)_{\lambda}$-weakly expansive condition (2.2). Suppose that the following conditions are satisfied:

(i) $A(X) \subset S(X), A(X) \subset T(X)$, and $A(X)$ is a closed subset of $\left(X, p_{b}^{s}\right)$;

(ii) $A$ is an injective and $A$ and $T$ are weakly compatible.

Then $A, S$, and $T$ have a unique common fixed point in $X$. 
Proof Let $x_{0} \in X$. Repeating the proof of Theorem 2.1, we know that there exists a sequence $\left\{A x_{n}\right\}$ in $X$ such that $A x_{2 n}=S x_{2 n+1}$ and $A x_{2 n+1}=T x_{2 n+2}$, for $n=0,1,2, \ldots$

We will complete the proof in three steps.

Step 1. We prove that $\lim _{n \rightarrow \infty} p_{b}\left(A x_{n}, A x_{n+1}\right)=0$.

Suppose first that $p_{b}\left(A x_{n}, A x_{n+1}\right)=0$ for some $n=n_{0}$. Then $A x_{n_{0}}=A x_{n_{0}+1}$. In the case that $n_{0}=2 k$, then $p_{b}\left(A x_{2 k}, A x_{2 k+1}\right)=0$ gives $p_{b}\left(A x_{2 k+1}, A x_{2 k+2}\right)=0$. Indeed, by (2.2), we have

$$
\begin{aligned}
0 & =g\left(\frac{p_{b}\left(A x_{2 k}, A x_{2 k+1}\right)}{\lambda^{2}}\right)=g\left(\frac{p_{b}\left(S x_{2 k+1}, T x_{2 k+2}\right)}{\lambda^{2}}\right) \\
& \geq h\left(M_{\lambda}\left(A x_{2 k+1}, A x_{2 k+2}\right)\right)+\phi\left(M_{\lambda}\left(A x_{2 k+1}, A x_{2 k+2}\right)\right),
\end{aligned}
$$

where

$$
\begin{aligned}
M_{\lambda}\left(A x_{2 k+1}, A x_{2 k+2}\right)= & \max \left\{\min \left\{p_{b}\left(A x_{2 k+1}, A x_{2 k+2}\right), \frac{0+p_{b}\left(A x_{2 k+1}, A x_{2 k+2}\right)}{2}\right\},\right. \\
& \left.\min \left\{p_{b}\left(A x_{2 k+1}, A x_{2 k+2}\right), \frac{p_{b}\left(A x_{2 k}, A x_{2 k+2}\right)}{\lambda}\right\}\right\} .
\end{aligned}
$$

Thus, $\phi\left(M_{\lambda}\left(A x_{2 k+1}, A x_{2 k+2}\right)\right)=0$, implies that $p_{b}\left(A x_{2 k+1}, A x_{2 k+2}\right)=0$. Similarly, if $n_{0}=$ $2 k+1$, then $p_{b}\left(A x_{2 k+2}, A x_{2 k+3}\right)=0$. Consequently, $p_{b}\left(A x_{n}, A x_{n+1}\right) \equiv 0$ for $n \geq n_{0}$. Hence, $\lim _{n \rightarrow \infty} p_{b}\left(A x_{n}, A x_{n+1}\right)=0$.

Now, suppose that $p_{b}\left(A x_{n}, A x_{n+1}\right)>0$, for each $n$. By (2.2), we have

$$
\begin{aligned}
g\left(\frac{p_{b}\left(A x_{2 n}, A x_{2 n+1}\right)}{\lambda^{2}}\right) & =g\left(\frac{p_{b}\left(S x_{2 n+1}, T x_{2 n+2}\right)}{\lambda^{2}}\right) \\
& \geq h\left(M_{\lambda}\left(A x_{2 n+1}, A x_{2 n+2}\right)\right)+\phi\left(M_{\lambda}\left(A x_{2 n+1}, A x_{2 n+2}\right)\right),
\end{aligned}
$$

where

$$
\begin{aligned}
M_{\lambda}\left(A x_{2 n+1}, A x_{2 n+2}\right)= & \max \left\{\operatorname { m i n } \left\{p_{b}\left(A x_{2 n+1}, A x_{2 n+2}\right),\right.\right. \\
& \left.\frac{p_{b}\left(A x_{2 n}, A x_{2 n+1}\right)+p_{b}\left(A x_{2 n+1}, A x_{2 n+2}\right)}{2}\right\}, \\
& \left.\min \left\{p_{b}\left(A x_{2 n+1}, A x_{2 n+2}\right), \frac{p_{b}\left(A x_{2 n+2}, A x_{2 n}\right)}{\lambda}\right\}\right\}>0 .
\end{aligned}
$$

If $\min \left\{p_{b}\left(A x_{2 n+1}, A x_{2 n+2}\right), \frac{p_{b}\left(A x_{2 n}, A x_{2 n+1}\right)+p_{b}\left(A x_{2 n+1}, A x_{2 n+2}\right)}{2}\right\}=\frac{p_{b}\left(A x_{2 n}, A x_{2 n+1}\right)+p_{b}\left(A x_{2 n+1}, A x_{2 n+2}\right)}{2}>0$, then we have $p_{b}\left(A x_{2 n}, A x_{2 n+1}\right) \leq p_{b}\left(A x_{2 n+1}, A x_{2 n+2}\right)$. It follows from (2.15) and the properties of $\phi, g, h$ that

$$
\begin{aligned}
g\left(p_{b}\left(A x_{2 n}, A x_{2 n+1}\right)\right) & \geq g\left(\frac{p_{b}\left(A x_{2 n}, A x_{2 n+1}\right)}{\lambda^{2}}\right) \\
& \geq h\left(M_{\lambda}\left(A x_{2 n+1}, A x_{2 n+2}\right)\right)+\phi\left(M_{\lambda}\left(A x_{2 n+1}, A x_{2 n+2}\right)\right) \\
& >g\left(M_{\lambda}\left(A x_{2 n+1}, A x_{2 n+2}\right)\right) \\
& \geq g\left(\frac{p_{b}\left(A x_{2 n}, A x_{2 n+1}\right)+p_{b}\left(A x_{2 n+1}, A x_{2 n+2}\right)}{2}\right) .
\end{aligned}
$$


Since $g$ is nondecreasing, we get $p_{b}\left(A x_{2 n}, A x_{2 n+1}\right)>p_{b}\left(A x_{2 n+1}, A x_{2 n+2}\right)$, which is a contradiction. Thus, $M_{\lambda}\left(A x_{2 n+1}, A x_{2 n+2}\right) \geq p_{b}\left(A x_{2 n+1}, A x_{2 n+2}\right)$. From (2.15), using the properties of $\phi, g, h$, we have

$$
g\left(p_{b}\left(A x_{2 n}, A x_{2 n+1}\right)\right) \geq g\left(\frac{p_{b}\left(A x_{2 n}, A x_{2 n+1}\right)}{\lambda^{2}}\right)>g\left(p_{b}\left(A x_{2 n+1}, A x_{2 n+2}\right)\right) .
$$

Hence, we deduce that, for each $n \in N, p_{b}\left(A x_{2 n+1}, A x_{2 n+2}\right)<p_{b}\left(A x_{2 n}, A x_{2 n+1}\right)$. Similarly, we can prove that $p_{b}\left(A x_{2 n}, A x_{2 n+1}\right)<p_{b}\left(A x_{2 n-1}, A x_{2 n}\right)$, for all $n \geq 1$. Therefore, $\left\{p_{b}\left(A x_{n}, A x_{n+1}\right)\right\}$ is a decreasing sequence of nonnegative real numbers. So, there exists $r \geq 0$ such that $\lim _{n \rightarrow \infty} p_{b}\left(A x_{n}, A x_{n+1}\right)=r$. Following the proof of Theorem 2.1, we know that the sequence $\left\{p_{b}\left(A x_{2 n}, A x_{2 n+2}\right)\right\}$ has a subsequence $\left\{p_{b}\left(A x_{n_{k}}, A x_{n_{k}+2}\right)\right\}$ which converges to a real number $\alpha \leq 2 \lambda r$. By (2.15), we get

$$
\begin{aligned}
g\left(\frac{p_{b}\left(A x_{2 n_{k}}, A x_{2 n_{k}+1}\right)}{\lambda^{2}}\right) & =g\left(\frac{p_{b}\left(S x_{2 n_{k}+1}, T x_{2 n_{k}+2}\right)}{\lambda^{2}}\right) \\
& \geq h\left(M_{\lambda}\left(A x_{2 n_{k}+1}, A x_{2 n_{k}+2}\right)\right)+\phi\left(M_{\lambda}\left(A x_{2 n_{k}+1}, A x_{2 n_{k}+2}\right)\right) .
\end{aligned}
$$

Letting $n_{k} \rightarrow \infty$ in the above inequality, using the properties of $\phi, g, h$, we can see that

$$
\begin{aligned}
g(r) & \geq g\left(\frac{r}{\lambda^{2}}\right) \geq h\left(\max \left\{\min \{r, r\}, \min \left\{r, \frac{\alpha}{\lambda}\right\}\right\}\right)+\phi\left(\max \left\{\min \{r, r\}, \min \left\{r, \frac{\alpha}{\lambda}\right\}\right\}\right) \\
& \geq h(r)+\phi(r),
\end{aligned}
$$

which implies that $r=0$. Therefore, $\lim _{n \rightarrow \infty} p\left(A x_{n}, A x_{n+1}\right)=0$.

Step 2. We now show that $\left\{A x_{n}\right\}$ is a $p_{b}$-Cauchy sequence.

Indeed, we first prove that $\lim _{m, n \rightarrow \infty} p_{b}\left(A x_{n}, A x_{m}\right)=0$. Since $\lim _{n \rightarrow \infty} p_{b}\left(A x_{n}, A x_{n+1}\right)=0$, it is sufficient to show that $\lim _{m, n \rightarrow \infty} p_{b}\left(A x_{2 n}, A x_{2 m}\right)=0$. Suppose on the contrary, then there exists $\varepsilon>0$ for which we can find two subsequences $\left\{A x_{2 n(k)}\right\}$ and $\left\{A x_{2 m(k)}\right\}$ of $\left\{A x_{2 n}\right\}$ such that $m(k)$ is the smallest index, for which $m(k)>n(k)>k, p_{b}\left(A x_{2 m(k)}, A_{2 n(k)}\right) \geq \varepsilon$, for every $k$. This means that $p_{b}\left(A x_{2 m(k)-2}, A_{2 n(k)}\right)<\varepsilon$.

Repeating to the proof of Theorem 2.1, we also have (2.8)-(2.11). By means of (2.2), we get

$$
\begin{aligned}
g\left(\frac{p_{b}\left(A x_{2 n(k)}, A x_{2 m(k)-1}\right)}{\lambda^{2}}\right) & =g\left(\frac{p_{b}\left(S x_{2 n(k)+1}, T x_{2 m(k)}\right)}{\lambda^{2}}\right) \\
& \geq h\left(M_{\lambda}\left(A x_{2 n(k)+1}, A x_{2 m(k)}\right)\right)+\phi\left(M_{\lambda}\left(A x_{2 n(k)+1}, A x_{2 m(k)}\right)\right),
\end{aligned}
$$

where

$$
\begin{aligned}
M_{\lambda}\left(A x_{2 n(k)+1}, A x_{2 m(k)}\right)= & \max \left\{\operatorname { m i n } \left\{p_{b}\left(A x_{2 n(k)+1}, A x_{2 m(k)}\right),\right.\right. \\
& \left.\frac{p_{b}\left(A x_{2 n(k)+1}, A x_{2 n(k)}\right)+p_{b}\left(A x_{2 m(k)}, A x_{2 m(k)-1}\right)}{2}\right\}, \\
& \left.\min \left\{p_{b}\left(A x_{2 n(k)+1}, A x_{2 m(k)}\right), \frac{p_{b}\left(A x_{2 m(k)}, A x_{2 n(k)}\right)}{\lambda}\right\}\right\} .
\end{aligned}
$$


Taking the lower limit as $k \rightarrow \infty$, using (2.8), (2.9), and (2.10), it is clear that

$$
\liminf _{k \rightarrow \infty} M_{\lambda}\left(A x_{2 n(k)+1}, A x_{2 m(k)}\right) \geq \max \left\{\min \left\{\frac{\varepsilon}{\lambda}, 0\right\}, \min \left\{\frac{\varepsilon}{\lambda}, \frac{\varepsilon}{\lambda}\right\}\right\}=\frac{\varepsilon}{\lambda} .
$$

Taking the upper limit as $k \rightarrow \infty$ in (2.16), using the properties of $\phi, g, h,(2.11)$, and (2.17), we obtain

$$
g\left(\frac{\varepsilon}{\lambda}\right) \geq g\left(\frac{\limsup _{n \rightarrow \infty} p_{b}\left(A x_{2 n(k)}, A x_{2 m(k)-1}\right)}{\lambda^{2}}\right) \geq h\left(\frac{\varepsilon}{\lambda}\right)+\phi\left(\frac{\varepsilon}{\lambda}\right),
$$

which implies that $\varepsilon=0$, a contradiction. Hence, we obtain $\lim _{m, n \rightarrow \infty} p_{b}\left(A x_{n}, A x_{m}\right)=0$, that is, $\left\{A x_{n}\right\}$ is a $p_{b}$-Cauchy sequence.

Step 3. We will show that $A, S$, and $T$ have a unique common fixed point.

Since $\left\{A x_{n}\right\}$ is a $p_{b}$-Cauchy sequence in $\left(X, p_{b}\right)$. Similar to the proof of Theorem 2.1, we know that there exists $x^{*} \in X$ such that $\lim _{n \rightarrow \infty} p_{b}^{s}\left(A x_{n}, x^{*}\right)=0$.

Since $A(X)$ is a closed set of $\left(X, p_{b}^{s}\right), A(X) \subset T(X)$, and $\lim _{n \rightarrow \infty} p_{b}^{s}\left(A x_{n}, x^{*}\right)=0$, we get $x^{*} \in A(X) \subset T(X)$. Hence, there exists $z_{1} \in X$ such that $T z_{1}=x^{*}$. This together with (2.2) ensures that

$$
\begin{aligned}
g\left(\frac{p_{b}\left(A x_{2 n}, T z_{1}\right)}{\lambda^{2}}\right) & =g\left(\frac{p_{b}\left(S x_{2 n+1}, T z_{1}\right)}{\lambda^{2}}\right) \\
& \geq h\left(M_{\lambda}\left(A x_{2 n+1}, A z_{1}\right)\right)+\phi\left(M_{\lambda}\left(A x_{2 n+1}, A z_{1}\right)\right),
\end{aligned}
$$

where

$$
\begin{aligned}
M_{\lambda}\left(A x_{2 n+1}, A z_{1}\right)= & \max \left\{\min \left\{p_{b}\left(A x_{2 n+1}, A z_{1}\right), \frac{p_{b}\left(A x_{2 n+1}, A x_{2 n}\right)+p_{b}\left(A z_{1}, T z_{1}\right)}{2}\right\},\right. \\
& \left.\min \left\{p_{b}\left(A x_{2 n+1}, A z_{1}\right), \frac{p_{b}\left(A z_{1}, A x_{2 n}\right)}{\lambda}\right\}\right\} .
\end{aligned}
$$

Taking the upper limit as $n \rightarrow \infty$ in (2.18), using the properties of $\phi, g, h,(2.13)$, and (2.14), we get

$$
\begin{aligned}
0= & g(0) \\
\geq & h\left(\max \left\{\min \left\{\frac{p_{b}\left(A z_{1}, T z_{1}\right)}{\lambda}, \frac{p_{b}\left(A z_{1}, T z_{1}\right)}{2}\right\}, \min \left\{\frac{p_{b}\left(A z_{1}, T z_{1}\right)}{\lambda}, \frac{p_{b}\left(A z_{1}, T z_{1}\right)}{\lambda^{2}}\right\}\right\}\right) \\
& +\phi\left(\max \left\{\min \left\{\frac{p_{b}\left(A z_{1}, T z_{1}\right)}{\lambda}, \frac{p_{b}\left(A z_{1}, T z_{1}\right)}{2}\right\}, \min \left\{\frac{p_{b}\left(A z_{1}, T z_{1}\right)}{\lambda}, \frac{p_{b}\left(A z_{1}, T z_{1}\right)}{\lambda^{2}}\right\}\right\}\right),
\end{aligned}
$$

which implies that $p\left(A z_{1}, T z_{1}\right)=0$. Hence, $A z_{1}=T z_{1}=x^{*}$. Similarly, since $x^{*} \in A(X) \subset S(X)$, there exists $z_{2} \in X$ such that $S z_{2}=x^{*}$, we have $A z_{2}=S z_{2}=x^{*}$. Hence, $A z_{1}=A z_{2}=x^{*}$. Since $A$ is an injective, we get $z_{1}=z_{2}$.

Let $z=z_{1}=z_{2}$. Then $A z=S z=T z=x^{*}$. Since $A$ and $T$ are weakly compatible, it is obvious that $A x^{*}=A A z=A T z=T A z=T x^{*}$. Then we can find by (2.2) that

$$
g\left(\frac{p_{b}\left(A z, A x^{*}\right)}{\lambda^{2}}\right)=g\left(\frac{p_{b}\left(S z, T x^{*}\right)}{\lambda^{2}}\right) \geq h\left(M_{\lambda}\left(A z, A x^{*}\right)\right)+\phi\left(M_{\lambda}\left(A z, A x^{*}\right)\right),
$$


where

$$
\begin{aligned}
M_{\lambda}\left(A z, A x^{*}\right)= & \max \left\{\min \left\{p_{b}\left(A z, A x^{*}\right), \frac{p_{b}\left(A x^{*}, A x^{*}\right)}{2}\right\},\right. \\
& \left.\min \left\{p_{b}\left(A z, A x^{*}\right), \frac{p_{b}\left(A x^{*}, A z\right)}{\lambda}\right\}\right\} \geq \frac{p_{b}\left(A x^{*}, A z\right)}{\lambda} .
\end{aligned}
$$

Thus,

$$
g\left(\frac{p_{b}\left(A z, A x^{*}\right)}{\lambda}\right) \geq g\left(\frac{p_{b}\left(A z, A x^{*}\right)}{\lambda^{2}}\right) \geq h\left(\frac{p_{b}\left(A x^{*}, A z\right)}{\lambda}\right)+\phi\left(\frac{p_{b}\left(A x^{*}, A z\right)}{\lambda}\right),
$$

which implies that $p_{b}\left(A z, A x^{*}\right)=0$. Thus, $A z=A x^{*}$. Since $A$ is an injective, we get $z=x^{*}$. Thus, $A z=S z=T z=z$ and $z$ is a common fixed point of $A, S$, and $T$.

Now, we prove the uniqueness of common fixed points of $A, S$, and $T$. Suppose that $x^{*}, y^{*} \in X$ such that $A x^{*}=S x^{*}=T x^{*}=x^{*}$ and $A y^{*}=S y^{*}=T y^{*}=y^{*}$. By means of (2.2), we have

$$
g\left(\frac{p_{b}\left(x^{*}, y^{*}\right)}{\lambda^{2}}\right)=g\left(\frac{p_{b}\left(S x^{*}, T y^{*}\right)}{\lambda^{2}}\right) \geq h\left(M_{\lambda}\left(A x^{*}, A y^{*}\right)\right)+\phi\left(A x^{*}, A y^{*}\right),
$$

where

$$
\begin{aligned}
M_{\lambda}\left(A x^{*}, A y^{*}\right)= & \max \left\{\min \left\{p_{b}\left(x^{*}, y^{*}\right), \frac{p_{b}\left(x^{*}, x^{*}\right)+p_{b}\left(y^{*}, y^{*}\right)}{2}\right\},\right. \\
& \left.\min \left\{p_{b}\left(x^{*}, y^{*}\right), \frac{p_{b}\left(x^{*}, y^{*}\right)}{\lambda}\right\}\right\} \geq \frac{p_{b}\left(x^{*}, y^{*}\right)}{\lambda} .
\end{aligned}
$$

Hence,

$$
g\left(\frac{p_{b}\left(x^{*}, y^{*}\right)}{\lambda}\right) \geq g\left(\frac{p_{b}\left(x^{*}, y^{*}\right)}{\lambda^{2}}\right) \geq h\left(\frac{p_{b}\left(x^{*}, y^{*}\right)}{\lambda}\right)+\phi\left(\frac{p_{b}\left(x^{*}, y^{*}\right)}{\lambda}\right),
$$

which implies that $p_{b}\left(x^{*}, y^{*}\right)=0$. Hence, $x^{*}=y^{*}$. This completes the proof.

Remark 2.2 Taking $g \equiv h$ in Theorem 2.2, we have the following corollary, which extends and generalizes Theorem 2.1 in [15] and Theorem 1 in [17].

Corollary 2.2 Let $\left(X, p_{b}\right)$ be a $p_{b}$-complete partial b-metric space, $A, S$, and $T: X \rightarrow X$ be three mappings. Suppose that the following conditions are satisfied:

(i) $A(X) \subset S(X), A(X) \subset T(X)$, and $A(X)$ is a closed subset of $\left(X, p_{b}^{s}\right)$;

(ii) $A$ is an injective and $A$ and $T$ are weakly compatible;

(iii) for all $x, y \in X$, we have

$$
g\left(\frac{p_{b}(S x, T y)}{\lambda^{2}}\right) \geq g\left(M_{\lambda}(A x, A y)\right)+\phi\left(M_{\lambda}(A x, A y)\right)
$$

where

$M_{\lambda}(A x, A y)=\max \left\{\min \left\{p_{b}(A x, A y), \frac{p_{b}(A x, S x)+p_{b}(A y, T y)}{2}\right\}, \min \left\{p_{b}(A x, A y), \frac{p_{b}(A y, S x)}{\lambda}\right\}\right\}, \phi, g$ are the same as in Definition 2.2.

Then $A, S$, and $T$ have a unique common fixed point in $X$. 
In the sequel, we will take an example to support our results of Theorem 2.2.

Example 2.3 Let $X=R^{+}$. Define a partial $b$-metric $p_{b}: X \times X \rightarrow R^{+}$by

$$
p_{b}(x, y)=(\max \{x, y\})^{2}, \quad \text { for all } x, y \in X .
$$

It is easy to see that $\left(X, p_{b}\right)$ is a $p_{b}$-complete partial $b$-metric space with $\lambda=2$. Let $A, S, T$ : $X \rightarrow X$ be defined by

$$
A x=x, \quad S x=T x=\frac{5}{2} x \sqrt{1+\frac{1}{1+x^{2}}}, \quad \text { for all } x \in X .
$$

Then it is easy to show that all the conditions (i)-(ii) of Theorem 2.2 are satisfied. Define $\phi, g, h: R^{+} \rightarrow R^{+}$by $g(t)=h(t)=t, \phi(t)=\frac{t}{1+t}$, for all $t \in R^{+}$. Without loss of generality, we assume that $x \leq y$. Then

$$
\begin{aligned}
\frac{p_{b}(S x, T y)}{4} & =\frac{\left(\max \left\{\frac{5}{2} x \sqrt{1+\frac{1}{1+x^{2}}}, \frac{5}{2} y \sqrt{1+\frac{1}{1+y^{2}}}\right\}\right)^{2}}{4}=\frac{\left(\frac{5}{2} y \sqrt{1+\frac{1}{1+y^{2}}}\right)^{2}}{4} \\
& =\frac{25}{16}\left(y^{2}+\frac{y^{2}}{1+y^{2}}\right) \geq y^{2}+\frac{y^{2}}{1+y^{2}}=p_{b}(A x, A y)+\phi\left(p_{b}(A x, A y)\right) .
\end{aligned}
$$

That is,

$$
g\left(\frac{p_{b}(S x, T y)}{\lambda^{2}}\right) \geq h\left(p_{b}(A x, A y)\right)+\phi\left(p_{b}(A x, A y)\right) \geq h\left(M_{\lambda}(A x, A y)\right)+\phi\left(M_{\lambda}(A x, A y)\right),
$$

for all $x, y \in X$, where $M_{\lambda}(A x, A y)=\max \left\{\min \left\{p_{b}(A x, A y), \frac{p_{b}(A x, S x)+p_{b}(A y, T y)}{2}\right\}, \min \left\{p_{b}(A x, A y)\right.\right.$, $\left.\frac{p_{b}(A y, S x)}{\lambda}\right\}$. Thus, all conditions of Theorem 2.2 are satisfied. Hence, $A, S$, and $T$ have a unique common fixed point $x=0$.

\section{An application}

In this section, we establish the existence theorem for the solutions of a class of system of integral equations.

Consider the system of integral equations

$$
\left\{\begin{array}{l}
x(t)=\int_{0}^{T} K(t, s) f_{1}(t, s, x(s)) d s+x_{0}(t) \\
x(t)=\int_{0}^{T} K(t, s) f_{2}(t, s, x(s)) d s+x_{0}(t)
\end{array}\right.
$$

for $t \in I=[0, T]$, where $T>0, K: I^{2} \rightarrow R^{+}$is a continuous function and $f_{1}, f_{2}: I^{2} \times R \rightarrow R$ are also continuous functions.

Let $X=C(I, R)$ be the set of all real continuous functions defined on $I$. We endowed $X$ with the partial $b$-metric

$$
p_{b}(x, y)=\max _{t \in I}|x(t)-y(t)|^{q}+a,
$$

for all $x, y \in X$, where $a \in R^{+}$and $q \geq 1$. It is not difficult to prove that $\left(X, p_{b}\right)$ is a $p_{b^{-}}$ complete partial $b$-metric space with coefficient $\lambda=2^{q-1}$. 
Now, we define $S$ and $T: X \rightarrow X$ by

$$
S x(t)=\int_{0}^{T} K(t, s) f_{1}(t, s, x(s)) d s+x_{0}(t), \quad T x(t)=\int_{0}^{T} K(t, s) f_{2}(t, s, x(s)) d s+x_{0}(t),
$$

for all $x \in X$. Then $x$ is a solution of (3.1) if and only if it is a common fixed point of $S$ and $T$. We shall prove the existence of common fixed point of $S$ and $T$ under certain conditions.

Theorem 3.1 Suppose that the following hypotheses hold:

(i) there exist a continuous function $G: I^{2} \rightarrow R^{+}$and $\psi \in \Psi$ such that

$$
\begin{aligned}
& \frac{K(t, s)\left[f_{1}(t, s, x(s))-f_{2}(t, s, y(s))\right]+\frac{a}{T}}{4^{q-1}} \\
& \quad \geq G(t, s) \psi\left(p_{b}(x, y), p_{b}(x, S x), p_{b}(y, T y), \frac{p_{b}(x, T y)}{2^{q-1}}, \frac{p_{b}(y, S x)}{2^{q-1}}\right),
\end{aligned}
$$

for all $t, s \in I$, where $K(t, s) f_{1}(t, s, x(s))+\frac{a}{T} \geq K(t, s) f_{2}(t, s, y(s))$, for all $t, s \in I$.

(ii) $\inf _{t \in I} \int_{0}^{T} G(t, s) d s \geq 1$.

Then the system of integral equations (3.1) has a solution $x^{*} \in X$.

Proof Let $\lambda=2^{q-1}$. From the conditions (i) and (ii), we have

$$
\begin{aligned}
& \max _{t \in I}|S x(t)-T y(t)|+a \\
\lambda^{2} & \\
& =\frac{\max _{t \in I}\left|\int_{0}^{T} K(t, s) f_{1}(t, s, x(s)) d s-\int_{0}^{T} K(t, s) f_{2}(t, s, y(s)) d s\right|+a}{\lambda^{2}} \\
& \geq \frac{\max _{t \in I}\left|\int_{0}^{T}\left[K(t, s)\left(f_{1}(t, s, x(s))-f_{2}(t, s, y(s))\right)+\frac{a}{T}\right] d s\right|}{\lambda^{2}} \\
& \geq \frac{\int_{0}^{T}\left[K(t, s)\left(f_{1}(t, s, x(s))-f_{2}(t, s, y(s))\right)+\frac{a}{T}\right] d s}{\lambda^{2}} \\
& \geq \int_{0}^{T} G(t, s) \psi\left(p_{b}(x, y), p_{b}(x, S x), p_{b}(y, T y), \frac{p_{b}(x, T y)}{\lambda}, \frac{p_{b}(y, S x)}{\lambda}\right) d s \\
& =\psi\left(p_{b}(x, y), p_{b}(x, S x), p_{b}(y, T y), \frac{p_{b}(x, T y)}{\lambda}, \frac{p_{b}(y, S x)}{\lambda}\right) \int_{0}^{T} G(t, s) d s \\
& \geq \psi\left(p_{b}(x, y), p_{b}(x, S x), p_{b}(y, T y), \frac{p_{b}(x, T y)}{\lambda}, \frac{p_{b}(y, S x)}{\lambda}\right),
\end{aligned}
$$

for all $x, y \in X$. Thus, for any $x, y \in X$, we get the inequality of Corollary 2.1. Hence, all the hypotheses of Corollary 2.1 are satisfied. Then $S$ and $T$ have a common fixed point $x^{*} \in X$, that is, $x^{*}$ is a solution of the system of integral equations (3.1).

The authors declare that they have no competing interests. 


\section{Acknowledgements}

The authors thank the editor and the referees for their valuable comments and suggestions. The research was supported by the National Natural Science Foundation of China $(11071108,11361042,11326099)$ and the Provincial Natural Science Foundation of Jiangxi, China (2010GZS0147, 20114BAB201007, 20142BAB211004, 20142BAB201007), and supported partly by the Provincial Graduate Innovation Foundation of Jiangxi, China (YC2012-B004).

\section{Received: 5 March 2014 Accepted: 12 November 2014 Published: 27 Nov 2014}

\section{References}

1. Matthews, SG: Partial metric topology. In: Proc. 8th Summer Conference on General Topology and Applications. Ann. New York Acad. Sci., vol. 728, pp. 183-197 (1994)

2. Altun, I, Sola, F, Simsek, H: Generalized contractions on partial metric spaces. Topol. Appl. 157, 2778-2785 (2010)

3. Ćirić, L, Samet, B, Aydi, H, Vetro, C: Common fixed points of generalized contractions on partial metric spaces and an application. Appl. Math. Comput. 218, 2398-2406 (2011)

4. Samet, B, Rajović, M, Lazović, R, Stojiljković, R: Common fixed-point results for nonlinear contractions in ordered partial metric spaces. Fixed Point Theory Appl. 2011, Article ID 71 (2011)

5. Aydi, H, Karapınar, E, Shatanawi, W: Coupled fixed point results for $(\psi, \varphi)$-weakly contractive condition in ordered partial metric spaces. Comput. Math. Appl. 62, 4449-4460 (2011)

6. Abbas, M, Nazir, T: Fixed point of generalized weakly contractive mappings in ordered partial metric spaces. Fixed Point Theory Appl. 2012, Article ID 1 (2012)

7. Kadelbury, Z, Nashine, HK, Radenović, S: Fixed point results under various contractive conditions in partial metric spaces. Rev. R. Acad. Cienc. Exactas Fís. Nat., Ser. A Mat. 107, 241-256 (2013)

8. Bakhtin, IA: The contraction principle in quasimetric spaces. In: Functional Analysis, vol. 30, pp. $26-37$ (1989)

9. Czerwik, S: Contraction mappings in b-metric spaces. Acta Math. Inform. Univ. Ostrav. 1, 5-11 (1993)

10. Shi, L, Xu, SY: Common fixed point theorems for two weakly compatible self-mappings in cone $b$-metric spaces. Fixed Point Theory Appl. 2013, 120 (2013)

11. Hussain, N, Parvaneh, V, Roshan, JR, Kadelburg, Z: Fixed points of cyclic weakly $(\psi, \varphi, L, A, B)$-contractive mappings in ordered b-metric spaces with applications. Fixed Point Theory Appl. 2013, 256 (2013)

12. Roshan, JR, Parvaneh, V, Sedghi, S, Shobkolaei, N, Shatanawi, W: Common fixed points of almost generalized $(\psi, \varphi)_{s}$-contractive mappings in ordered $b$-metric spaces. Fixed Point Theory Appl. 2013, 159 (2013)

13. Plebaniak, R: New generalized pseudodistance and coincidence point theorem in a $b$-metric space. Fixed Point Theory Appl. 2013, 270 (2013)

14. Azam, A, Mehmood, N, Ahmad, J, Redenović, S: Multivalued fixed point theorems in cone $b$-metric spaces. J. Inequal. Appl. 2013, $582(2013)$

15. Roshan, JR, Parvaneh, V, Altun, I: Some coincidence point results in ordered b-metric spaces and applications in a system of integral equations. Appl. Math. Comput. 226, 725-757 (2014)

16. Shukla, S: Partial $b$-metric spaces and fixed point theorems. Mediterr. J. Math. 11,703-711 (2014)

17. Mustafa, Z, Roshan, JR, Parvaneh, V, Kadelburg, Z: Some common fixed point results in ordered partial $b$-metric spaces. J. Inequal. Appl. 2013, 562 (2013)

18. Jungck, G: Common fixed points for noncontinuous nonself maps on nonmetric spaces. Far East J. Math. Sci. 4 199-215 (1996)

19. Karapınar, E, Shahi, P, Kaur, J, Bhatia, SS: Generalized $(\xi, \alpha)$-expansive mappings and related fixed-point theorems. J. Inequal. Appl. 2014, Article ID 22 (2014)

20. Nashine, HK, Golubović, Z, Kadelburg, Z: Modified $\psi$-contractive mappings in ordered metric spaces and applications. Fixed Point Theory Appl. 2013, 203 (2013)

21. Aage, CT, Salunke, JN: Some fixed point theorems for expansion onto mappings on cone metric spaces. Acta Math. Sin. Engl. Ser. 27(6), 1101-1106 (2011)

22. Kumar, S, Garg, SK: Expansion mapping theorems in metric spaces. Int. J. Contemp. Math. Sci. 4(36), 1749-1758 (2009)

23. Kumar, S: Common fixed points theorems for expansion mappings in various spaces. Acta Math. Hung. 118, 9-28 (2008)

10.1186/1029-242X-2014-475

Cite this article as: Zhu et al.: Common fixed point theorems for generalized expansive mappings in partial $b$-metric spaces and an application. Journal of Inequalities and Applications 2014, 2014:475

\section{Submit your manuscript to a SpringerOpen ${ }^{\circ}$ journal and benefit from:}

- Convenient online submission

- Rigorous peer review

- Immediate publication on acceptance

- Open access: articles freely available online

- High visibility within the field

- Retaining the copyright to your article 